\title{
Portal vein resection in advanced pancreatic adenocarci- noma: is it worth the risk?
}

\author{
Katharina Marsoner · Rainer Langeder · Dora Csengeri · Gottfried Sodeck · Hans Jörg Mischinger · \\ Peter Kornprat
}

Received: 3 February 2016 / Accepted: 25 May 2016 / Published online: 30 June 2016

(C) The Author(s) 2016. This article is available at SpringerLink with Open Access

\begin{abstract}
Summary
Introduction Portal vein resection represents a viable add-on option in standard pancreaticoduodenectomy for locally advanced ductal pancreatic adenocarcinoma, but is often underused as it may set patients at additional risk for perioperative and postoperative morbidity and mortality. We aimed to review our long-term experience to determine the additive value of this intervention for locally advanced pancreatic adenocarcinoma.

Patients and methods Single, university surgical center audit over a 13-year period; cohort comprised 221 consecutive patients undergoing pancreatic resection; in $47(21 \%)$ including portal vein resection. Predictors for short- and long-term survival were assessed via multivariate logistic and Cox regression.

Results Baseline and perioperative characteristics were similar between the two groups. However, overall skin-to-skin times, intraoperative transfusion requirements as the need for medical inotropic support were higher in patients undergoing additional portal vein resection $(p<0.0001 ; p=0.001$ and $p=0.03)$. Postoperative complication rates were 34 vs. $35 \%$ ( $p=$ 0.89 ), 14 patients ( $5 \%$ vs. $11 \% ; p=0.18$ ) died in-hospital. An American Society of Anesthesiologists Score $>2$ was the only independent predictor for in-hospital mortality (OR 10.66, 95 \% CI 1.24-91.30). Follow-up
\end{abstract}

Katharina Marsoner and Rainer Langeder contribute equally.

K. Marsoner, MD · R. Langeder, MD · D. Csengeri, MD ·

Prof. H. J. Mischinger, MD - Prof. P. Kornprat, MD (ه)

Department of General Surgery, Medical University of Graz,

Auenbruggerplatz 29, $8036 \mathrm{Graz}$, Austria

E-Mail: peter.kornprat@medunigraz.at

G. Sodeck, MD

Department of Emergency Medicine, Medical University of

Vienna, Vienna, Austria was complete in $99.5 \%$, one-year survival was $59 \%$ vs. $70 \%$ and five-year overall survival $15 \%$ vs. $12 \%$ with and without portal vein resection, respectively (Log rank: $p=0.25$ ). For long-term outcome, microvascular invasion (HR 2.03, $95 \%$ CI 1.10-3.76) and preoperative weight loss (HR 2.17, $95 \%$ CI 1.31-3.58) were independent predictors.

Conclusion Despite locally advanced disease, patients who underwent portal vein resection had no worse perioperative and overall survival than patients with lower staging and standard pancreaticoduodenectomy only. Therefore, the feasibility of portal vein resection should be evaluated in every potential candidate at risk.

Keywords Pancreatic ductal adenocarcinoma - Pancreaticoduodenectomy - Portal vein resection - Perioperative outcome $\cdot$ Long-term survival

\section{Introduction}

Complete surgical resection of pancreatic ductal adenocarcinoma (PDAC) represents the key factor for survival despite advances in chemo- and radiochemotherapy. In the 1970s, Fortner first described a radical en bloc surgical resection of venous portal branches and surrounding tissues [1]. Despite this approach might improve survival in locally advanced PDAC, surgeons often are concerned about this technique in fear of the potential additional risk for perioperative and postoperative morbidity and mortality [2-5]. We aimed therefore to review our long-term experience to determine the additional value of portal venous resection in locally advanced PDAC. 


\section{Materials and methods}

\section{Study procedures}

Single center, university surgical center audit over a 13-year period; cohort compromised 221 consecutive patients (112 females; median age 67 years [IQR interquartile range(60-72)]) undergoing pancreatic resection for diagnosis of PDAC; in 47 (21\%) portal vein resection (PVR) has been performed as an add-on due to locally advanced disease making a R0 resection impossible without additional resection of portal venous tissue. Baseline and perioperative risk factors were recorded; the ASA (American Association of Anesthesiologist's) Score was applied to estimate the perioperative risk [6]. All eligible patients underwent a standardized preoperative screening program including detailed physical examination including tumor markers carcino embryotic antigen (CEA), CA 19-9 (carbohydrate antigen) and liver function tests; preoperative oncological staging comprised positron emission tomography (PET) scan, endoscopic retrograde cholangiopancreaticography (ERCP) and/or magnetic resonance cholangiopancreaticography (MRCP) and esophagogastroduodenoscopy (EGD). If not already performed at initial diagnosis, all patients received a multidetector row computed tomography (MDCT) with pancreas protocol. Prior to definite decision for surgery all patients were admitted to the institutional tumor board including surgeons, oncologists, histopathologists, and radiologists. The institutional review board approved the study and waived the need for patient consent.

\section{Portal vein resection technique}

Depending on the primary tumor location, either a standard or pylorus-preserving pancreaticoduodenectomy, distal pancreatectomy with splenectomy or total pancreatectomy was carried out in all cases. The biliodigestive anastomoses were connected with $5-0$ or $6-0$ double layer single sutures; the biliodigestive anastomoses were performed routinely via end-to-side pancreaticojejunostomy and hepaticojejunostomy and were protected by means of internal drainages.

Portal vein resection was performed in all cases of radiologic and/or intraoperative diagnosis of tumorous adherence or infiltration of the portal venous wall, allowing a potential R0 resection with additional resection of the portal venous segment. Prior to cross-clamp of the portal venous branches all patients received intravenously 5000 international units (IU) heparin. In case that tumor infiltration of the venous wall was less than one-third of the circumference, this particular segment was resected in an elliptic fashion and reconstructed with a Gore-tex ${ }^{\circledR}$ patch; otherwise the affected vein segment was resected completely: reconstruction of the portal axis was achieved via a tension-free end-to-end anastomosis with a continuous running $6-0$ polypropylene suture or via a Gore-tex ${ }^{\circledR}$ tube graft when a longer vein segment had to be resected. Heparinization was not reversed at the end of the procedure and continued intravenously until oral prescription of unlimited aspirin or clopidogrel upon discretion of the surgeon. Intraabdominal drainages were placed routinely in all patients before closure of the abdominal wall.

\section{Histopathological examination}

Intraoperative rapid frozen section diagnosis was part of the standard protocol and according to the intraoperative histopathologic diagnosis, resection was extended until negative margins could be obtained or the surgical strategy was switched to a total pancreatectomy. In definitive histopathological examination, a hematoxylin-eosin staining according to standard procedures was performed, in cases with portal venous involvement, the grade of vascular infiltration was differentiated between adhesion, adventitial, media, or transmural infiltration.

\section{Follow-up protocol}

Postoperative morbidity was classified according to Clavien and Dindo (CDC) [7]. A drainage cholangiography was performed in all patients before discharge to exclude anastomotic leakage. Patients underwent clinical, laboratory, and radiological (MDCT) followup 3, 6, and 12 months postoperatively. Long-term mortality data were obtained from the Austrian National Cancer registry [8].

\section{Statistical analysis}

If not otherwise indicated, continuous variables were reported as median and interquartile range; categorical data were reported as count and percentages. Categorical variables were compared with Fisher's exact text or the $\chi^{2}$ test, as appropriate; metric variables were compared with the Wilcoxon test. A multivariate logistic regression model was applied to assess the strongest independent risk factor for intrahospital mortality. Results of the statistic regression model are given as the odds ratio (OR) and $95 \%$ confidence interval (CI). A resampling model with 1000 replications was then used to confirm CI. Regression diagnostics and overall model fit were performed according to standard procedures, providing Hosmer-Lemeshow tests for calibration and $\mathrm{C}$ statistics for discrimination of the final model. Overall survival and progressionfree survival were calculated according to the method of Kaplan and Meier. A Cox regression model was used for multivariate and univariate analysis to identify predictors for worse long-term outcome. A twosided $p$-value $<0.05$ was considered statistically sig- 
original article

Table 1 Baseline and preoperative demographic patient's characteristics

\begin{tabular}{|c|c|c|c|c|}
\hline Characteristics & Overall $(n=221)$ & Without portal vein resection $(n=174)$ & With portal vein resection $(n=47)$ & Two-sided $p$ value \\
\hline \multicolumn{5}{|l|}{ Demographic data } \\
\hline Age (years) & $67(60-72)$ & $67(60-73)$ & $66(60-71)$ & 0.25 \\
\hline Female sex & $112(51 \%)$ & $89(51 \%)$ & $23(49 \%)$ & 0.87 \\
\hline BMI & $24(22-28)$ & $25(22-28)$ & $23(22-26)$ & 0.09 \\
\hline Age $>70$ years & $75(34 \%)$ & $62(36 \%)$ & $13(27 \%)$ & 0.39 \\
\hline \multicolumn{5}{|l|}{ Chronic health conditions (\%) } \\
\hline Alcohol abuse & $19(9)$ & $14(8)$ & $5(11)$ & 0.57 \\
\hline Nicotine abuse & $41(19)$ & $32(19)$ & $9(19)$ & 1.00 \\
\hline Chronic pancreatitis & $27(13)$ & $22(13)$ & $5(11)$ & 1.00 \\
\hline Diabetes & $60(27)$ & $48(28)$ & $12(26)$ & 0.86 \\
\hline Liver/renal cysts & $9(4)$ & $7(4)$ & $2(4)$ & 1.00 \\
\hline ASA $>2$ & $133(62)$ & $109(64)$ & $24(52)$ & 0.17 \\
\hline Gastrointestinal comorbidity & $43(19)$ & $33(19)$ & $10(21)$ & 0.68 \\
\hline Cardiovascular comorbidity & $132(60)$ & $108(62)$ & $24(51)$ & 0.18 \\
\hline Pulmonary comorbidity & $27(12)$ & $24(14)$ & $3(6)$ & 0.21 \\
\hline Other comorbidities & $71(34)$ & $56(34)$ & $15(34)$ & 1.00 \\
\hline $\begin{array}{l}\text { Extrapancreatic malignancy (current } \\
\text { and/or anamnestic) }\end{array}$ & $36(16)$ & $28(16)$ & $8(17)$ & 1.00 \\
\hline \multicolumn{5}{|l|}{ Preoperative symptoms (\%) } \\
\hline Emesis & $22(10)$ & $17(10)$ & $5(12)$ & 0.78 \\
\hline Abdominal pain & $107(50)$ & $83(49)$ & $24(56)$ & 0.50 \\
\hline Jaundice & $64(30)$ & $50(29)$ & $14(32)$ & 0.72 \\
\hline Acute pancreatitis & $27(13)$ & $20(12)$ & $7(16)$ & 0.44 \\
\hline Diarrhea & $16(8)$ & $13(8)$ & $3(7)$ & 1.00 \\
\hline Weight loss & $66(30)$ & $51(30)$ & $15(32)$ & 0.86 \\
\hline \multicolumn{5}{|l|}{ Preoperative diagnostics (\%) } \\
\hline СT & $176(84)$ & $137(84)$ & $39(83)$ & 0.83 \\
\hline MRI & 122(59) & $89(55)$ & $33(70)$ & 0.09 \\
\hline PET-CT & $65(31)$ & $45(28)$ & $20(46)$ & 0.07 \\
\hline ERCP & $85(40)$ & $63(38)$ & $22(47)$ & 0.31 \\
\hline PTCD & $17(8)$ & $14(8)$ & $3(6)$ & 1.00 \\
\hline \multicolumn{5}{|l|}{ Radiol. tumor size (\%) } \\
\hline$<3 \mathrm{~cm}$ & $82(49)$ & $61(35)$ & $21(52)$ & 0.23 \\
\hline $3-5 \mathrm{~cm}$ & $59(36)$ & $42(33)$ & $17(29)$ & 0.10 \\
\hline $5-7 \mathrm{~cm}$ & $17(10)$ & $16(13)$ & $1(3)$ & 0.08 \\
\hline$>7 \mathrm{~cm}$ & $6(4)$ & $5(4)$ & $1(3)$ & 0.21 \\
\hline Not available & $63(29)$ & & & \\
\hline Biopsy preop & $25(12)$ & $18(11)$ & $7(15)$ & 0.45 \\
\hline Radiological portal venous infiltration & $32(14)$ & $13(7)$ & $19(47)$ & $<0.0001$ \\
\hline
\end{tabular}

nificant and SPSS 22.0 for Windows (IBM Inc, Somers, NY, USA) was used for all statistical analyses.

\section{Results}

Over a 13-year period, 221 consecutive patients (112 females; median age 67 years [IQR 60-72]) were subject to pancreatic resection for diagnosis of PDAC at our institution; in 47 (21\%) portal vein resection was deemed necessary as an add-on due to locally advanced disease.

Detailed demographics and cohort comparisons are given in Tables 1 and 2: Baseline and perioperative characteristics were similar between the two groups without statistical significance except preoperative finding of portal venous infiltration in radiological imaging $(p<0.0001)$ Obviously due to the additional procedure, overall skin-to skin times (median 361 [IQR 311-422] vs. 307 [IQR 236-361] minutes in pa- 
Table 2 Intra-and postoperative patient's characteristics

\begin{tabular}{|c|c|c|c|c|}
\hline Characteristics & Overall $(n=221)$ & Without portal vein resection $(n=174)$ & With portal vein resection $(n=47)$ & Two-sided $p$ value \\
\hline \multicolumn{5}{|l|}{ Intraoperative data } \\
\hline Skin-to-skin time (min) & $318(248-375)$ & $307(236-361)$ & $361(311-422)$ & $<0.0001$ \\
\hline Kausch Whipple (\%) & $77(35)$ & $58(33)$ & $19(40)$ & 0.37 \\
\hline $\begin{array}{l}\text { Pylorus preserving } \\
\text { pancreaticoduodenectomy (\%) }\end{array}$ & $65(30)$ & $54(31)$ & $11(23)$ & 0.31 \\
\hline Central resection (\%) & $1(1)$ & $1(1)$ & 0 & 0.60 \\
\hline Distal pancreatectomy (\%) & $47(21)$ & $40(23)$ & $7(15)$ & 0.23 \\
\hline Total pancreatectomy (\%) & $31(14)$ & $21(12)$ & $10(21)$ & 0.11 \\
\hline Splenectomy (\%) & $68(32)$ & $51(29)$ & $17(36)$ & 0.48 \\
\hline Internal drainage (\%) & $122(58)$ & $94(54)$ & $28(60)$ & 0.74 \\
\hline Intraoperative blood transfusion (\%) & $108(62)$ & $74(55)$ & $34(85)$ & 0.001 \\
\hline Intraoperative inotropic support (\%) & $37(19)$ & $23(16)$ & $14(32)$ & 0.03 \\
\hline \multicolumn{5}{|l|}{ Postoperative patient's characteristics } \\
\hline ICU stay (days) & $3(2-5)$ & $3(2-4.5)$ & $3(2-5)$ & 0.11 \\
\hline In-hospital stay (days) & $24(18-31)$ & $23(17-31)$ & $25(20-31)$ & 0.23 \\
\hline Postoperative blood transfusion (\%) & $34(18)$ & $9(5)$ & $5(11)$ & 0.18 \\
\hline In-hospital mortality (\%) & $14(6)$ & $9(5)$ & $5(11)$ & 0.18 \\
\hline
\end{tabular}

Table 3 Histopathological details

\begin{tabular}{ll|l|l|l|}
\hline $\begin{array}{l}\text { Characteristics } \\
\text { Histopathological data }\end{array}$ & Overall $(n=221)$ & Without portal vein resection $(n=174)$ & With portal vein resection $(n=47)$ & Two-sided $p$ value \\
\hline Pancreatitis (\%) & $62(30)$ & $51(32)$ & $11(24)$ & 0.36 \\
\hline Perineural invasion (\%) & $49(23)$ & $36(21)$ & $13(28)$ & 0.32 \\
\hline Lymphovascular invasion (\%) & $105(52)$ & $79(50)$ & $26(59)$ & 0.31 \\
\hline R0 [dorsal margin <1 mm (\%)] & $106(60)$ & $87(62)$ & $19(50)$ & 0.24 \\
\hline R0 (\%) & $180(87 \%)$ & $141(87 \%)$ & $39(89)$ & 0.76 \\
\hline Lymph node ratio & $0.16(0.06-0.33)$ & $0.16(430.05-0.33)$ & $0.13(0.07-0.29)$ & 0.43 \\
\hline
\end{tabular}

Table 4 Details of portal vein infiltration

\begin{tabular}{|l|l|}
\hline Depth of portal vein infiltration & $n(\%)$ \\
\hline Adventitia & $6(13)$ \\
\hline Media & $5(11)$ \\
\hline Transmural & $11(23)$ \\
\hline Tumor adherence & $15(32)$ \\
\hline No specification & $10(21)$ \\
\hline
\end{tabular}

tient with and without PVR, respectively), transfusion requirements ( 85 vs. $55 \%$ of patients) as the need for medical inotropic support (32 vs. 16\%) were higher in patients undergoing additional portal vein resection $(p<0.0001 ; p=0.001$ and $p=0.03)$. Detailed histopathological results were given in Tables 3 and 4 .

Postoperative complication rates according to Clavien and Dindo (CDC) were 34 vs. $35 \%(p=0.89)$, and 14 patients (5 vs. $11 \% ; p=0.18$ ) died in-hospital. An ASA Score $>2$ was the only independent predictor for in-hospital mortality in logistic regression analysis (OR 10.66, $95 \%$ CI 1.24-91.30; H/L = 0.73, c-statistics: $p=0.79$ ). The strong influence of an ASA Score $>2$ on in-hospital mortality could be confirmed in bootstrap analysis (95\% CI 1.04-22.12; Table 5).
Table 5 Results of univariate logistic regression analysis of in-hospital mortality concerning selected potential predictors'

\begin{tabular}{|l|l|l|}
\hline Risk factor & $\mathrm{OR}^{\star}\left(95 \% \mathrm{Cl}^{\star *}\right)$ & Two-sided $p$-value \\
\hline Sex & $0.35(0.08-1.53)$ & 0.16 \\
\hline Age $>70$ years & $1.43(0.36-5.68)$ & 0.61 \\
\hline Alcohol abuse & $1.34(0.21-8.49)$ & 0.76 \\
\hline Diabetes mellitus & $0.26(0.05-1.47)$ & 0.13 \\
\hline ASA Score $>$ II & $10.66(1.24-91.30)$ & 0.03 \\
\hline Preop. weight loss & $0.80(0.18-3.52)$ & 0.77 \\
\hline Preop. jaundice & $1.08(0.25-4.66)$ & 0.92 \\
\hline Preop. pancreatitis & $1.05(0.17-6.37)$ & 0.96 \\
\hline ERCP preop & $0.67(0.16-2.79)$ & 0.58 \\
\hline UICC $>$ II & $3.90(0.58-26.25)$ & 016 \\
\hline PVR & $2.31(0.57-9.34)$ & 0.24 \\
\hline
\end{tabular}

$O R$ Odds ratio, $C /$ Confidence interval, $A S A$ Score Anesthesiologic risk score of the American society of anesthesiology, ERCP Endoscopic retrograde cholangiopancreaticography, UICC Union Internationale contre la Cancer; clinical tumor stage, PVR portal vein resection 
Table 6 Comparison of patients with and without portal vein resection by follow-up characteristics

\begin{tabular}{|c|c|c|c|c|}
\hline Follow up characteristics & Overall cohort & Without portal vein resection $(n=174)$ & With portal vein resection $(n=47)$ & Two-sided $p$-value \\
\hline Follow-up (months) & $12(6-26)$ & $12(6-28)$ & $11(7-20)$ & 0.52 \\
\hline \multicolumn{5}{|l|}{ Status at follow up (\%) } \\
\hline NED & $46(21)$ & $36(21)$ & $10(21)$ & 0.93 \\
\hline AWD & $31(14)$ & $27(16)$ & $4(9)$ & 0.22 \\
\hline DOD & $140(63)$ & $107(48)$ & 330 & 0.27 \\
\hline DOC & $3(1)$ & & & \\
\hline Lost of follow-up & $1(0.5)$ & & & \\
\hline Chemotherapy & $126(57)$ & $98(56)$ & $27(60)$ & 0.94 \\
\hline Radiotherapy & $3(1)$ & $2(1)$ & $1(2)$ & 0.61 \\
\hline Progression-free survival (months) & $7(4-16)$ & $8(4-15)$ & $7(4-16)$ & 0.43 \\
\hline
\end{tabular}

Table 7 Uni-and multivariate predictors for survival (Cox regression)

\begin{tabular}{|c|c|c|}
\hline Predictor & Univariate $(\mathrm{HR} / 95 \% \mathrm{Cl})$ & Multivariate (HR/95\% Cl) \\
\hline Sex & $0.82(95 \mathrm{Cl} 0.60-1.14)$ & 1.09 (95 Cl 0.71-1.68) \\
\hline Age $>70$ years & 1.31 (95 Cl 0.92-1.86) & 1.39 (95 Cl 0.87-2.22) \\
\hline Diabetes mellitus & 1.01 (95 Cl 0.75-1.56) & 1.66 (95 Cl 1.04-2.63) \\
\hline $\begin{array}{l}\text { Cardiovascular co- } \\
\text { morbidity }\end{array}$ & 0.89 (95 Cl 0.63-1.24) & 0.62 (95 Cl 0.39-0.99) \\
\hline $\begin{array}{l}\text { Preoperative symp- } \\
\text { tomatic }\end{array}$ & 1.25 (95 Cl 0.85-1.82) & 0.93 (95 Cl 0.59-1.48) \\
\hline $\begin{array}{l}\text { Preoperative weight } \\
\text { loss }\end{array}$ & 1.45 (95 Cl 1.00-2.09) & 2.17 (95 Cl 1.31-3.58) \\
\hline Whipple procedure & 0.56 (95 Cl 0.39-0.79) & 0.48 (95 Cl 0.31-0.75) \\
\hline Portal vein resection & 1.18 (95 Cl 0.79-1.76) & 0.61 (95 Cl 0.33-1.15) \\
\hline Free margin (R0) & 0.90 (95 Cl 0.62-1.13) & 0.78 (95 Cl 0.50-1.22) \\
\hline Vascular invasion & 1.62 (95 Cl 1.12-2.35) & 2.03 (95 Cl 1.10-3.76) \\
\hline $\begin{array}{l}\text { Lymphovascular } \\
\text { invasion }\end{array}$ & 1.63 (95 Cl 1.15-2.32) & 1.59 (95 Cl 1.02-2.45) \\
\hline Perineural invasion & 1.52 (95 Cl 1.02-2.24) & 1.53 (95 Cl 0.95-2.47) \\
\hline Organ infiltration & 1.70 (95 Cl 1.17-2.47) & 1.04 (95 Cl 0.63-1.71) \\
\hline Tumor grading & 1.79 (95 Cl 1.35-2.37) & 1.78 (95 Cl 1.23-3.57) \\
\hline Chemotherapy & 0.16 (95 Cl 0.05-0.53) & 0.05 (95 Cl 0.01-0.27) \\
\hline
\end{tabular}

Follow-up was complete in $99.5 \%$ and comprised 4393 patients-months of follow-up (for details Table 6): 1-year and 5-year overall survival were 59 and $15 \%$ vs. 70 and $12 \%$ with and without PVR, respectively (Log-rank: $p=0.25$; Fig. 1 ).

For mid-term and long-term outcome, microvascular invasion (hazard ratio, HR 2.03, $95 \%$ CI 1.10-3.76), preoperative weight loss (HR 2.17, $95 \%$ CI 1.31-3.58), lymphovascular invasion (HR 1.59, $95 \%$ CI 1.03-2.45) and tumor grading (HR $1.7895 \%$ CI 1.23-2.57) were independent predictors for mortality during followup; but not portal vein resection (HR 0.61, 95\% CI 0.33-1.15); Whipple procedure (HR $0.48 \quad 95 \%$ CI 0.01-0.75) and chemotherapy (HR $0.0595 \%$ CI 0.01-0.27) were associated with lower risk of death during follow-up (Table 7).

\section{Discussion}

As to our institutional long-term experience portal vein resection represents a viable add-on option in pancreatic surgery for locally advanced PDAC: compared to patients without locally advanced disease, short- and long-term results are acceptable without significant differences.

In recent decades, infiltration of portal venous and/or mesenteric venous branches has ceased to be synonymous with inoperability $[1,9]$. Portal vein resection represents therefore the last option for patients with locally advanced disease to receive potential curative surgery [1-5, 10-12] after diagnosis of PDAC.

However, there is still an ongoing debate if pancreatic surgery with vascular resection and reconstruction increases the incidence of postoperative complications and morbidity [2-5, 9-12]. Our postoperative complication rates according to Clavien and Dindo (CDC) were 34 vs. $35 \%$ ( $p=0.89$ ), and 14 patients $(5$ vs. $11 \% ; p=0.18$ ) died in-hospital, which are in line with previously reported data for stand-alone pancreaticoduodenectomy [13, 14]. This particular finding might be explained by the fact that our hepatobiliary surgeons undergo extensive training in the vascular surgical techniques. As a result, only two patients required revision for bleeding; in one patient it was probably due to development of a pancreatic fistula with subsequent erosion hemorrhage; in the remaining patient, the bleeding occurred within $24 \mathrm{~h}$ postoperatively.

Further in contrast to others, all patients receive continuous intravenous heparin in the early postoperative period followed by platelet inhibitors in the late postoperative period [15]. Indeed, only one patient experienced portal vein thrombosis together with abscess formation a few days after operation and an unfavorable outcome. Computed tomography follow-up has proven the patency of portal venous reconstructions in all discharged patients. 


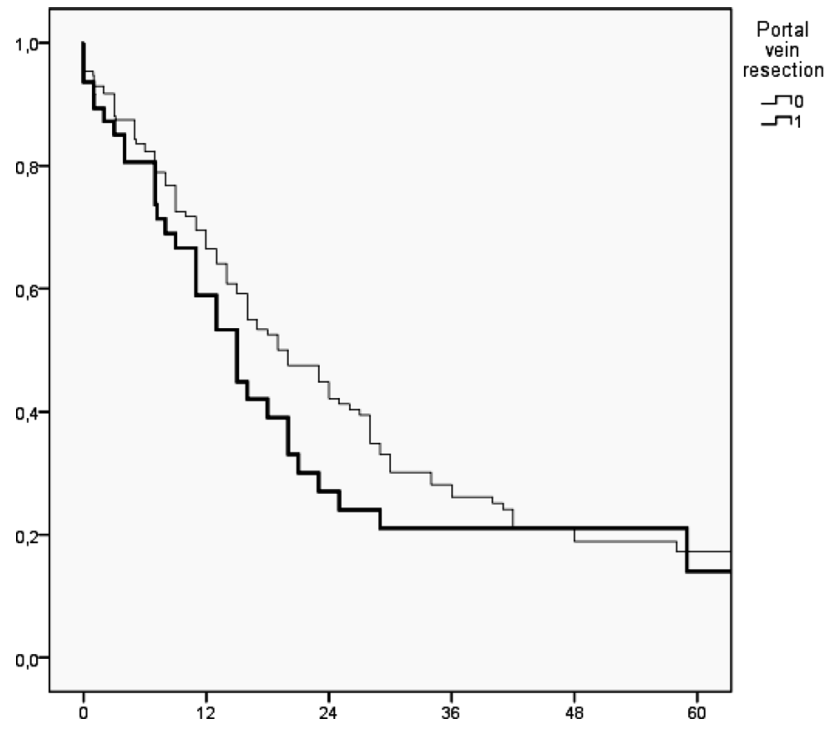

Fig. 1 Kaplan-Meier plot of overall survival in patients with and without portal vein resection'

Current evidence is mixed regarding the risk of the surgical procedure itself and postoperative morbidity and mortality; although numerous publications propose different scoring systems to predict perioperative mortality and morbidity for pancreatic resection, no clinically established preoperative scoring system is available at this point $[16,17]$. As to our observation, portal vein resection has no impact upon immediate survival; an ASA Score $>2$ was the only independent predictor for in-hospital mortality in logistic regression analysis (OR 10.66, $95 \%$ CI 1.24-91.30) has confirmed via bootstrap modelling. Indeed, all patients but one who died during their intrahospital stay were scored into ASA class 3 or 4 . As to our opinion, this well-established anesthesiologic score gives an excellent picture of patient's preoperative general health conditions not only predicting the anesthesiologic risk alone but also the perioperative risk for pancreatic resections.

Median overall survival in our cohort did not differ between the cohorts with and without additional portal vein resection. This finding is in line with other publications in the field and would suggest that this intervention is not worth the effort for locally advanced PDAC [10-12, 18]. However, from the oncologic point of view, it is the only chance for patients with locally advanced PDAC to achieve similar survival rates compared to those patients with lower staging [10-12, 18]. As to our observation, besides extensive preoperative weight loss (HR 2.17, $95 \%$ CI 1.31-3.58) reflecting the advanced stage of disease, microvascular invasion reflecting the aggressive tumor biology (HR 2.03, $95 \%$ CI 1.10-3.76) was the strongest independent predictor for long-term survival.

To summarize, portal vein resection as an addon in pancreatic resection does not adversely impact immediate, short- and long-term outcome in locally advanced PDAC as preoperative risk factors determine. The anesthesiologic ASA score gives an excellent picture of patient's preoperative general health conditions not only predicting the anesthesiologic risk alone but also the perioperative risk for pancreatic resections. The feasibility of portal vein resection should therefore be evaluated in every potential candidate at risk.

\section{Study limitations}

Some limitations of the present study have to be acknowledged. Although we could demonstrate that a ASA Score $>2$ is a strong predictor of postoperative outcome and not the surgical intervention itself, sample size is modest when it comes to random variability, resulting in wide confidence intervals. To limit this potential bias we performed a bootstrap analysis confirming our initial results. However, our findings from a retrospective single-center study will ideally need confirmation in multicenter studies. Second, we modified slightly the definitions of the international study group (ISGPF) for postoperative complications [19] due to the retrospective nature of study design: We have only documented clinically significant pancreatic fistulas; a standardized analysis of drainage liquid for amylase and lipase is not part of our routine protocol. Furthermore, in our cohort, we did not assess the incidence of delayed gastric emptying, as to our institutional protocol, all patients receive a nasogastral tube and prokinetic drugs (metoclopramide and/or domperidone) in the early postoperative period. Residual confounding by patient management at the operating theater and intensive care unit, however, may be still present. Such confounders are impossible to control in an observational retrospective study design. But considering overall death, our main end point, this bias appears to be negligible as patient-related data were retrieved from a validated nationwide Austrian Cancer Database [8].

Conflict of interest K. Marsoner, R. Langeder, D. Csengeri, P. Kornprat, G. Sodeck and H.J. Mischinger declare that they have no competing interests.

Open Access This article is distributed under the terms of the Creative Commons Attribution 4.0 International License (http://creativecommons.org/licenses/by/4.0/), which permits unrestricted use, distribution, and reproduction in any medium, provided you give appropriate credit to the original author(s) and the source, provide a link to the Creative Commons license, and indicate if changes were made.

\section{References}

1. Kim DK, Cubilla A, Turnbull A, Pahnke LD, Fortner JG, Shils ME. Regional pancreatectomy; en bloc pancreatic, portal vein and lymph node resection. Ann Surg. 1977;186(1):42-50.

2. Fuhrman GM,LeachSD, etal. Rationaleforenblocresection in the treatment of panreatic adenocarcinoma adherent to 
the superior mesenteric-portal vein confluence. Ann Surg. 1996;223(2):154-62.

3. Bachellier P, Nakano H, Oussoultzoglou PD, et al. Is pancreaticoduodenenctoymy with mesentericoportal venous resection safe and worthwile? Am J Surg. 2001;82:120-9.

4. Riediger H, Makowiec F, Fischer E, Adam U, Hopt UT. Postoperative morbidity and long-term survival after pancreaticoduodenectomy with superior mesenterico-portal vein resection. J Gastrointest Surg. 2006;10(8):1106-15.

5. Yekebas EF, Bodoevski D, Cataldegirmen G, et al. En bloc vascular resection for local advanced pancreatic malignancies infiltrating major blood vessels: perioperative outcome and long-term survival in 136 patients. Ann Surg. 2008;247(2):300-9.

6. http://www.asahq.org/resources/clinical-information/ asa-physical-status-classification-system. Accessed: 16th of June 2016.

7. Dindo D, Demartines N, Clavien PA. Classification of surgical complications: a new proposal with evaluation in a cohort of 6336 patients and results of a survey. Ann Surg. 2004;240:205-13.

8. Austrian National cancer registry. http://www.statistik. at/web_de/statistiken/menschen_und_gesellschaft/ gesundheit/krebserkrankungen/bauchspeicheldruese/ index.html.

9. Ramacciato G, Mercantini P, Petrucciani N, et al. Does portal-superior mesenteric vein invasion still indicate irresectability for pancreatic carcinoma? Ann Surg Oncol. 2009;16(4):817-25.

10. Yekebas EF, Bogoevski D, Cataldegirmen G, et al. En bloc vascular resection for locally advanced pancreatic malignancies infiltrating major blood vessels: perioperative outcome and long-term survival in 136 patients. Ann Surg. 2008;247(2):300-9.
11. Ravikumar R, Sabin C, Abu Hilal M, et al. UK vascular resection in pancreatic cancer study group.: portal vein resection in borderline resectable pancreatic cancer: a united kingdom multicenter study. J Am Coll Surg. 2014;218(3):401-11.

12. CastleberryAW, WhiteRR, DeLaFuenteSG, etal. Theimpact of vascular resection on early postoperative outcomes after pancreaticoduodenectomy: an analysis of the American College ofSurgeons National Surgical Quality Improvement Program database. Ann Surg Oncol. 2012;19(13):4068-77.

13. Cameron JL, Riall TS, Coleman J, Belcher KA. One thousand consecutive pancreaticoduodenectomies. Ann Surg. 2006;244(1):10-5.

14. Andrén-Sandberg A, Neoptolemos JP. Resection for pancreatic cancer in the new millenium. Pancreatology. 2002;2(5):431-9.

15. Chandrasegaram MD, Eslick GD, Lee W, et al. Anticoagulation policy after venous resection with a pancreatectomy a systematic review. HPB (Oxford). 2014;16(8):691-8.

16. Braga M, Capretti G, Pecorelli N, et al. A prognostic score to predict major complications after pancreaticoduodenectomy. Ann Surg. 2011;254(5):702-7.

17. Uzunoglu FG, Reeh M, Vettorazzi E, et al. Preoperative pancreatic resection (PREPARE) Score: a prospective multi-center based morbidity risk score. Ann Surg. 2014;260(5):857-63.

18. Gong Y, Zhang L, He T, et al. Pancreaticoduodenenctomy combined with vascular resection and reconstruction for patients with locally advanced pancreatic cancer: a multicenter retrospective analysis. PLoS ONE. 2013;8(8):e70340 doi:10.1371/journal.pone.0070340.

19. Bassi C, Dervenis C, Butturini G, et al. Postoperative pancreatic fistula: in international study group (ISPGS) definition. Surgery. 2005;138:8-13. 